\title{
The Efficacy of Subgingivally Delivered Andrographis Paniculata Gel to Treat Patients with Periodontitis
}

\author{
Thitiwan Teparat-Burana ${ }^{1}$, Chalita Samarnsotthiwong ${ }^{2}$, Julalux Kasetsuwan ${ }^{3}$, \\ Mullika Sirirat ${ }^{4}$ and Pleumchitt Rojanapanthu ${ }^{5}$ \\ ${ }^{1,3,4}$ Department of Oral Medicine and Periodontology, Faculty of Dentistry, Mahidol University, \\ Bangkok, Thailand \\ ${ }^{2}$ Dental Department, Panomsarakam Hospital, Chachengsao, Thailand \\ ${ }^{5}$ Department of Pharmacy, Faculty of Pharmacy, Mahidol University, Bangkok, 10400, Thailand
}

Correspondence should be addressed to: Thitiwan Teparat-Burana; thitiwan.tep@mahidol.ac.th

Received date: 19 September 2013; Accepted date: 10 March 2014; Published date: 30 April 2015

Academic Editor: Mário Júnior Taba

Copyright (C) 2015. Thitiwan Teparat-Burana, Chalita Samarnsotthiwong, Julalux Kasetsuwan, Mullika Sirirat and Pleumchitt Rojanapanthu . Distributed under Creative Commons CC-BY 4.0

\begin{abstract}
This study compared the clinical effects of scaling and root planing (SRP) after the adjunctive subgingival administration of Andrographis paniculata (AP) gel or placebo (PB) gel in a 6-month clinical trial for patients with chronic periodontitis. The study was a splitmouth, single-blind, randomized and controlled clinical trial that compared two treatment modalities (SRP+PB and SRP+AP) in single-rooted teeth with a probing depth (PD) of $5 \mathrm{~mm}$ or more. The clinical parameters, including PD, clinical attachment level (CAL), plaque index (PI), gingival index (GI), bleeding on probing (BOP), and radiographic examinations were recorded at baseline, 3 months and 6 months. The results showed that both treatment groups significantly improved with regard to clinical parameters $(p<0.05)$; however, the between-group differences were mostly insignificant $(p>0.05)$, although SRP $+A P$ showed a greater PD reduction than SRP+PB $(p<0.05)$ between baseline and 3 months. In conclusion, the adjunctive use of AP gel significantly reduces PD and significantly improves attachment level.
\end{abstract}

Keywords: Andrographis paniculata gel, chronic periodontitis, non-surgical therapy

\section{Introduction}

Chronic periodontitis is an inflammatory disease of the tooth-supporting tissue. The primary etiological agent of this disease is the bacteria in dental plaque.
Microorganisms and their products destroy the periodontal tissues (gingiva, cementum, alveolar bone, and periodontal ligament) resulting in a loss of connective tissue attachment, pocket formation and alveolar bone resorption, the last of which

Cite this Article as: Thitiwan Teparat-Burana, Chalita Samarnsotthiwong, Julalux Kasetsuwan, Mullika Sirirat and Pleumchitt Rojanapanthu (2015), " The Efficacy of Subgingivally Delivered Andrographis Paniculata Gel to Treat Patients with Periodontitis " Journal of Research and Practice in Dentistry, 
causes tooth mobility and tooth loss. Periodontal therapy seeks to slow or halt disease progression, regenerate the periodontal tissue, and prevent disease recurrence (Haffajee and Socransky, 1994). Thus, preventing and treating chronic periodontitis can decrease tooth loss and promote good overall health.

The goals of an effectively treating chronic periodontitis are to arrest inflammatory processes via the removal of dental plaque, and to establish a local environment and microflora that are compatible with good periodontal health. Clinical outcomes to determine the success of periodontal treatment include reduced probing depth (PD), increased clinical attachment level (CAL), and reduced bleeding on probing (BOP). This treatment begins with nonsurgical therapy, which includes plaque control as well as scaling and root planing (SRP). Surgical therapy may be performed to gain an additional improvement after a successful non-surgical therapy. Considerable evidence supports the plaque control combined with SRP as an essential and effective component of the therapeutic measures for arresting periodontal disease (Badersten et al., 1990; Isidor and Karring, 1986; Pihlstrom et al., 1981; Knowles et al., 1980; Hughes and Caffesse, 1978; Listgarten et al., 1978). Nevertheless, certain factors can limit clinical and microbiological responses; these factors include pocket depth, root morphology, tooth position, and bacterial ability to invade periodontal tissues or dentinal tubules (Knowles et al., 1980; Rabbani et al., 1981; Waerhaug, 1978; Caffesse et al., 1986; Adriaens et al., 1988; Saglie et al., 1982).

The adjunctive use of chemical agents is another part of non-surgical therapy. Controlled-release antibiotic therapy has been introduced as an adjunct to enhance the efficacy of these non-surgical therapies. Many commercially available products exist, such as tetracycline fiber, metronidazole gel (Elyzol ${ }^{\circledR}$ ), ${ }^{\dagger}$ chlorhexidine chips (PerioChip ${ }^{\circledR}$ ), $\S$ and doxycycline polymer (Atridox ${ }^{\circledR}$ ). ${ }^{\top}$ However, these options are expensive because they must be imported from other countries. Thus, controlled-release drug systems using active components from traditional herbs have been developed. One of the most interesting plants is Andrographis paniculata Nees (AP), a medicinal herb that is widely found and cultivated in tropical and subtropical areas of Asia, including Thailand. AP contains a large quantity of bitters, which are primarily diterpenoid lactone compounds (Jewvachdamrongkul et al., 1987). The interesting pharmacological activities of AP for periodontal therapy are its antibacterial activity against Porphyromonas gingivalis and its anti-inflammatory and immunostimulatory activities (Amornchat et al., 1991; Anju et al., 1993; Meenatchisundaram et al., 2009). Several studies have shown that AP gel can be used effectively as an adjunct to SRP to treat periodontitis (Rassameemasmaung et al., 1998; Atsawasuwan et al., 1998; Boonchaipanichwatana, 2001; Sirirat and Rojanapanthu, 2003; Thawornrungroj et al., 2011).

This study compared the clinical effects of SRP with the adjunctive subgingival administration of AP or PB gels to treat patients with chronic periodontitis in a 6month clinical trial.

\section{Materials and Methods}

The Ethical Review Committee for Research in Human Subjects, Ministry of Public Health, Thailand approved the study protocol (No. 61/2009). All patients were provided with both verbal and written information regarding the study and included in the trial only after providing informed consent.

\section{Patient Selection}

A total of 35 patients diagnosed with chronic periodontitis (aged 35 years or older) were recruited from the Section of Periodontics, Department of Oral Medicine and Periodontology, Faculty of Dentistry, Mahidol University. The inclusion criteria were: 1) the presence of at least two singlerooted teeth in each quadrant with a PD of at least $5 \mathrm{~mm}$ and BOP; 2) radiographic evidence of alveolar bone loss; and 3) no 
periodontal treatments for at least 6 months prior to the study. Patients were excluded from participation if they 1) had diabetes; 2) had a systemic disease requiring daily medication; 3 ) had a history of antibiotic use over the previous 6 months; or 4) were pregnant or lactating.

\section{Gel Preparations}

The Faculty of Pharmacy, Mahidol University, Bangkok, Thailand created the preparations of $\mathrm{AP}$ and $\mathrm{PB}$ gel bases. The $\mathrm{AP}$ and $\mathrm{PB}$ gels were stored at $4^{\circ} \mathrm{C}$ throughout the study.

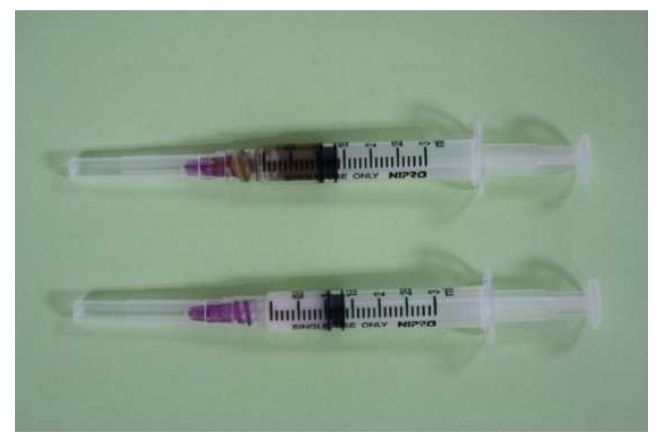

Figure 1: The AP (top) and PB gels (bottom

\section{Clinical Procedures}

This study used a split-mouth, single-blind controlled clinical trial to compare two treatment modalities: $\mathrm{SRP}+\mathrm{PB}$ and $\mathrm{SRP}+\mathrm{AP}$. Each modality was applied to the teeth in each quadrant based on a randomized list using a computer-generated table. The two remaining quadrants were using SRP alone.

An intra-examiner calibration was performed prior to the study, achieving intraclass correlation coefficients (ICCs) of $\geq 0.9$. All clinical parameters were measured at 6 sites around each tooth; however, only the two sites with the deepest PDs, CALs, or both (from different teeth within the same quadrant) were selected for the study. The clinical parameters included $\mathrm{PD}, \mathrm{CAL}$, the plaque index (PI; Silness \& Löe, 1964), the gingival index (GI; Löe \& Silness, 1963), BOP (Ainamo \& Bay, 1975), and tooth mobility (Miller, 1943).

One trained examiner recorded all clinical parameters and administered all radiographic examinations (using the parallel technique) at baseline, 3 months and 6 months. After the baseline examination, oral hygiene instructions were provided to the patients to perform self-plaque control using the Modified Bass method for brushing with either dental flossing or an interdental brush for interdental cleansing. The participants were asked to abstain from using antibacterial mouthwash for the entire study. Every tooth was mechanically debrided with an ultrasonic scaler (MiniPiezon, EMS ${ }^{\circledR}$, Ivoclar Vivadent, Schaan, Principality of Liechtenstein) and hand instruments (Gracey curettes, HuFriedy ${ }^{\circledR}$, Chicago, IL, USA) until the subgingival root surface was hard and smooth. Local anesthesia was used as necessary for patient comfort. In the $\mathrm{SRP}+\mathrm{PB}$ and SRP+AP groups, the periodontal pockets were gently irrigated with $2 \mathrm{ml}$ of $0.9 \%$ sterile saline in a syringe fitted with a 21-gauge blunted needle after SRP. Then, the PB or AP gels were gently applied subgingivally into the periodontal pockets around the selected teeth (Figure 2). A sufficient amount of gel was administered to fill each periodontal pocket to the gingival margin. The excess was removed using sterile cotton pellets. Strict guidance was provided to the patients not to rinse, drink, or eat for 1 hour after the gel was applied. This gel application was repeated 1, 2, and 3 weeks following the baseline. All clinical parameters and radiographic examinations 
were re-examined at 3 and 6 months after 1 week of gel administration. The AP or PB gels were also re-administered at the 3month re-examination. An examiner (CS) who was masked to the type of treatment received recorded all clinical parameters and performed SRP, whereas other operators (MS \& JK) administered the AP and PB gels. The patients were motivated to use an oral hygiene regimen for plaque control throughout the study.

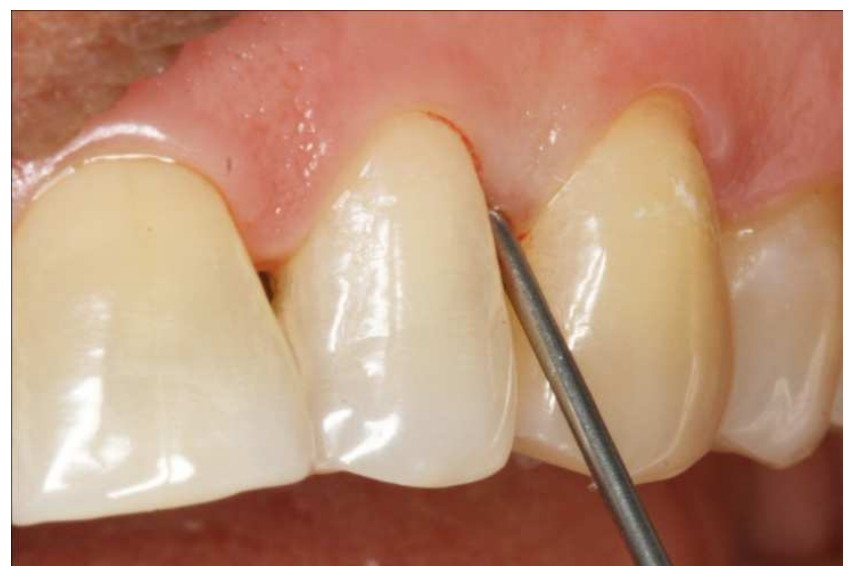

Figure 2: The PB or AP gels were gently loaded subgingivally into the periodontal pocket

\section{Data Analyses}

Data analyses were performed using PASW Statistics, version 18.0 (SPSS Inc., Chicago, IL, USA). The significance threshold was $\alpha=0.05$ for all tests. The intra-examiner reliability was calculated using ICCs. Between-treatment group comparisons of PD, CAL; the PI and the GI were analyzed using the Mann-Whitney U test. Withintreatment group comparisons and the differences between each time interval were analyzed using Friedman's test and Wilcoxon's signed-rank test. In addition, between-treatment group comparisons of BOP were assessed using the Chi-square test. Cochran's Q test was used to analyze within-treatment group comparisons of BOP, followed by pairwise comparisons using McNemar's test.

\section{Results}

A total of 35 participants with chronic periodontitis (13 men and 22 women, aged 35 to 73 years; mean age $45.31 \pm 9.16$ years) were enrolled in the study. A total of 526 sites were collected from 235 teeth. The study group consisted of four former smokers and five current smokers. No participants were excluded during the study. No side effects were noted in any of the participants, although some patients complained of a bitter taste after the AP gel application.

No significant differences were found between the groups with regard to any of the baseline clinical parameters except for the PI (Table 1\&2). 
Table 1: Mean patient clinical parameters

\begin{tabular}{|c|c|c|c|c|c|c|c|}
\hline \multirow{2}{*}{$\begin{array}{c}\text { Particip } \\
\text { ant }\end{array}$} & \multirow[t]{2}{*}{ Parameters } & \multicolumn{3}{|c|}{$\begin{array}{c}\mathrm{SRP}+\mathrm{PB} \\
(\mathrm{n}=271)\end{array}$} & \multicolumn{3}{|c|}{$\begin{array}{c}\mathrm{SRP}+\mathrm{AP} \\
(\mathrm{n}=292)\end{array}$} \\
\hline & & Baseline & 3 months & 6 months & Baseline & 3 months & 6 months \\
\hline 1 & $\begin{array}{c}\mathrm{PD}(\mathrm{mm}) \\
\text { CAL }(\mathrm{mm}) \\
\text { PI } \\
\text { GI } \\
\text { BOP }(\%)\end{array}$ & $\begin{array}{c}5.60 \pm 089 \\
7.00 \pm 1.58 \\
2.20 \pm 0.84 \\
2.00 \pm 0.00 \\
100.0\end{array}$ & $\begin{array}{c}3.80 \pm 1.15 \\
6.00 \pm 1.87 \\
0.40 \pm 0.55 \\
0.80 \pm 1.09 \\
40.0\end{array}$ & $\begin{array}{c}3.00 \pm 0.71 \\
5.20 \pm 1.30 \\
0.80 \pm 0.45 \\
0.40 \pm 0.89 \\
20.0\end{array}$ & $\begin{array}{c}6.67 \pm 1.53 \\
7.00 \pm 1.00 \\
1.67 \pm 0.58 \\
2.67 \pm 0.58 \\
100.0\end{array}$ & $\begin{array}{c}4.67 \pm 1.16 \\
6.33 \pm 1.53 \\
1.00 \pm 1.00 \\
2.00 \pm 0.00 \\
100.0\end{array}$ & $\begin{array}{c}3.67 \pm 1.16 \\
5.33 \pm 1.53 \\
1.33 \pm 0.58 \\
0.00 \pm 0.00 \\
0.0\end{array}$ \\
\hline 2 & $\begin{array}{c}\text { PD (mm) } \\
\text { CAL (mm) } \\
\text { PI } \\
\text { GI } \\
\text { BOP }(\%)\end{array}$ & $\begin{array}{c}5.20 \pm 0.45 \\
5.60 \pm 0.89 \\
2.00 \pm 0.00 \\
2.00 \pm 0.00 \\
100.0\end{array}$ & $\begin{array}{c}3.20 \pm 0.45 \\
4.20 \pm 1.09 \\
1.80 \pm 0.45 \\
2.00 \pm 0.00 \\
100.0\end{array}$ & $\begin{array}{c}3.20 \pm 0.45 \\
4.20 \pm 1.09 \\
1.40 \pm 0.89 \\
1.60 \pm 0.89 \\
80.0\end{array}$ & $\begin{array}{c}5.50 \pm 0.71 \\
5.50 \pm 0.71 \\
2.00 \pm 0.00 \\
1.50 \pm 0.71 \\
50.0\end{array}$ & $\begin{array}{c}3.50 \pm 0.71 \\
3.50 \pm 0.71 \\
2.00 \pm 0.00 \\
1.50 \pm 0.71 \\
50.0\end{array}$ & $\begin{array}{c}3.00 \pm 0.00 \\
3.00 \pm 0.00 \\
2.00 \pm 0.00 \\
1.50 \pm 0.71 \\
50.0\end{array}$ \\
\hline 3 & $\begin{array}{c}\text { PD (mm) } \\
\text { CAL (mm) } \\
\text { PI } \\
\text { GI } \\
\text { BOP }(\%)\end{array}$ & $\begin{array}{c}5.33 \pm 0.58 \\
5.33 \pm 0.58 \\
2.00 \pm 1.00 \\
1.67 \pm 0.58 \\
66.7\end{array}$ & $\begin{array}{c}4.33 \pm 0.58 \\
4.67 \pm 1.16 \\
1.00 \pm 1.00 \\
1.33 \pm 1.16 \\
66.7\end{array}$ & $\begin{array}{c}3.33 \pm 0.58 \\
3.67 \pm 1.16 \\
0.33 \pm 0.58 \\
1.33 \pm 1.16 \\
66.7\end{array}$ & $\begin{array}{c}6.00 \pm 0.00 \\
6.00 \pm 0.00 \\
1.00 \pm 0.00 \\
1.50 \pm 0.71 \\
50.0\end{array}$ & $\begin{array}{c}4.50 \pm 0.71 \\
4.50 \pm 0.71 \\
2.00 \pm 0.00 \\
2.00 \pm 0.00 \\
100.0\end{array}$ & $\begin{array}{c}3.00 \pm 0.00 \\
3.50 \pm 0.71 \\
1.50 \pm 0.71 \\
0.00 \pm 0.00 \\
0.0\end{array}$ \\
\hline 4 & $\begin{array}{c}\text { PD (mm) } \\
\text { CAL (mm) } \\
\text { PI } \\
\text { GI } \\
\text { BOP }(\%)\end{array}$ & $\begin{array}{c}5.00 \pm 0.00 \\
5.25 \pm 0.50 \\
1.75 \pm 0.50 \\
1.50 \pm 0.58 \\
50.0\end{array}$ & $\begin{array}{c}4.25 \pm 0.50 \\
5.25 \pm 1.89 \\
0.75 \pm 0.50 \\
2.00 \pm 0.00 \\
100.0\end{array}$ & $\begin{array}{c}4.25 \pm 0.50 \\
5.25 \pm 1.89 \\
1.00 \pm 0.00 \\
0.50 \pm 1.00 \\
25.0\end{array}$ & $\begin{array}{c}5.53 \pm 0.64 \\
6.47 \pm 0.92 \\
1.93 \pm 0.46 \\
1.93 \pm 0.26 \\
93.3\end{array}$ & $\begin{array}{c}4.13 \pm 0.64 \\
6.13 \pm 0.83 \\
0.93 \pm 0.70 \\
1.47 \pm 0.74 \\
60.0\end{array}$ & $\begin{array}{c}3.87 \pm 0.64 \\
5.87 \pm 0.99 \\
0.13 \pm 0.35 \\
1.40 \pm 0.83 \\
60.0\end{array}$ \\
\hline 5 & $\begin{array}{c}\text { PD }(\mathrm{mm}) \\
\text { CAL }(\mathrm{mm}) \\
\text { PI } \\
\text { GI } \\
\text { BOP }(\%)\end{array}$ & $\begin{array}{c}5.50 \pm 0.55 \\
5.67 \pm 0.52 \\
1.50 \pm 0.55 \\
1.83 \pm 0.41 \\
83.3\end{array}$ & $\begin{array}{c}4.00 \pm 0.00 \\
4.17 \pm 0.41 \\
1.17 \pm 0.41 \\
1.00 \pm 0.89 \\
33.3\end{array}$ & $\begin{array}{c}3.83 \pm 0.41 \\
4.00 \pm .63 \\
0.50 \pm 0.84 \\
1.00 \pm 0.89 \\
33.3\end{array}$ & $\begin{array}{c}7.00 \pm 1.27 \\
8.00 \pm 2.37 \\
1.33 \pm 0.52 \\
1.67 \pm 0.52 \\
66.7\end{array}$ & $\begin{array}{c}4.67 \pm 1.37 \\
5.67 \pm 2.50 \\
1.33 \pm 0.82 \\
0.83 \pm 0.75 \\
16.7\end{array}$ & $\begin{array}{c}4.33 \pm 1.21 \\
5.50 \pm 2.17 \\
0.00 \pm 0.00 \\
1.17 \pm 0.98 \\
50.0\end{array}$ \\
\hline 6 & $\begin{array}{c}\text { PD }(\mathrm{mm}) \\
\text { CAL }(\mathrm{mm}) \\
\text { PI } \\
\text { GI } \\
\text { BOP }(\%) \\
\end{array}$ & $\begin{array}{c}5.33 \pm 0.58 \\
5.33 \pm 0.58 \\
2.00 \pm 0.00 \\
2.00 \pm 0.00 \\
100.0\end{array}$ & $\begin{array}{c}4.33 \pm 1.53 \\
5.00 \pm 1.73 \\
1.00 \pm 1.00 \\
2.00 \pm 0.00 \\
100.0\end{array}$ & $\begin{array}{c}4.33 \pm 1.53 \\
5.00 \pm 1.73 \\
0.67 \pm 0.58 \\
0.67 \pm 1.16 \\
33.3 \\
\end{array}$ & $\begin{array}{c}5.17 \pm 0.41 \\
6.00 \pm 1.55 \\
2.00 \pm 0.00 \\
1.33 \pm 0.52 \\
33.3 \\
\end{array}$ & $\begin{array}{c}3.17 \pm 0.98 \\
4.17 \pm 0.98 \\
1.83 \pm 0.41 \\
1.00 \pm 1.09 \\
50.0\end{array}$ & $\begin{array}{c}3.17 \pm 0.75 \\
4.17 \pm 1.47 \\
0.83 \pm 0.75 \\
0.50 \pm 0.84 \\
16.7\end{array}$ \\
\hline 7 & $\begin{array}{c}\text { PD (mm) } \\
\text { CAL (mm) } \\
\text { PI } \\
\text { GI } \\
\text { BOP }(\%)\end{array}$ & $\begin{array}{c}5.00 \pm 0.00 \\
5.00 \pm 0.00 \\
1.33 \pm 0.58 \\
1.33 \pm 0.58 \\
33.3\end{array}$ & $\begin{array}{c}3.33 \pm 0.58 \\
3.33 \pm 0.58 \\
0.67 \pm 0.58 \\
0.33 \pm 0.58 \\
0.0\end{array}$ & $\begin{array}{c}3.33 \pm 0.58 \\
3.33 \pm 0.58 \\
0.00 \pm 0.00 \\
0.67 \pm 1.16 \\
33.3\end{array}$ & $\begin{array}{c}5.50 \pm 0.76 \\
5.50 \pm 0.76 \\
2.00 \pm 0.00 \\
1.63 \pm 0.52 \\
62.5\end{array}$ & $\begin{array}{c}3.50 \pm 0.93 \\
3.50 \pm 0.93 \\
0.63 \pm 0.52 \\
1.25 \pm 1.04 \\
62.5\end{array}$ & $\begin{array}{c}3.38 \pm 0.92 \\
3.88 \pm 0.84 \\
0.38 \pm 0.52 \\
1.38 \pm 0.92 \\
62.5\end{array}$ \\
\hline 8 & $\begin{array}{c}\text { PD }(\mathrm{mm}) \\
\text { CAL }(\mathrm{mm}) \\
\text { PI } \\
\text { GI } \\
\text { BOP }(\%)\end{array}$ & $\begin{array}{c}5.00 \pm 0.00 \\
5.20 \pm 0.45 \\
2.20 \pm 0.45 \\
2.00 \pm 0.00 \\
100.0\end{array}$ & $\begin{array}{c}2.80 \pm 1.30 \\
3.80 \pm 1.09 \\
1.20 \pm 0.45 \\
1.60 \pm 0.55 \\
60.0\end{array}$ & $\begin{array}{c}2.80 \pm 1.30 \\
3.80 \pm 1.09 \\
2.00 \pm 0.00 \\
1.40 \pm 0.55 \\
40.0\end{array}$ & $\begin{array}{c}6.20 \pm 1.48 \\
4.90 \pm 0.88 \\
2.60 \pm 0.52 \\
2.00 \pm 0.00 \\
100.0\end{array}$ & $\begin{array}{c}3.30 \pm 1.06 \\
2.90 \pm 0.74 \\
1.40 \pm 0.69 \\
1.50 \pm 0.53 \\
50.0\end{array}$ & $\begin{array}{c}2.90 \pm 0.99 \\
2.60 \pm 0.69 \\
1.20 \pm 0.79 \\
1.30 \pm 0.68 \\
40.0\end{array}$ \\
\hline
\end{tabular}




\section{Mean patient clinical parameters (Cont.)}

\begin{tabular}{|c|c|c|c|c|c|c|c|}
\hline \multirow{2}{*}{$\begin{array}{c}\text { Particip } \\
\text { ant }\end{array}$} & \multirow[t]{2}{*}{ Parameters } & \multicolumn{3}{|c|}{$\begin{array}{c}\mathrm{SRP}+\mathrm{PB} \\
(\mathrm{n}=271)\end{array}$} & \multicolumn{3}{|c|}{$\begin{array}{c}\mathrm{SRP}+\mathrm{AP} \\
(\mathrm{n}=292)\end{array}$} \\
\hline & & Baseline & 3 months & 6 months & Baseline & 3 months & 6 months \\
\hline 9 & $\begin{array}{c}\text { PD }(\mathrm{mm}) \\
\text { CAL }(\mathrm{mm}) \\
\text { PI } \\
\text { GI } \\
\text { BOP }(\%)\end{array}$ & $\begin{array}{c}5.79 \pm 0.98 \\
6.50 \pm 1.35 \\
1.93 \pm 0.27 \\
2.00 \pm 0.00 \\
100.0\end{array}$ & $\begin{array}{c}3.14 \pm 1.17 \\
5.57 \pm 1.65 \\
1.29 \pm 0.61 \\
1.00 \pm 1.04 \\
50.0\end{array}$ & $\begin{array}{c}2.64 \pm 0.75 \\
5.07 \pm 1.64 \\
1.21 \pm 0.43 \\
1.07 \pm 0.99 \\
50.0\end{array}$ & $\begin{array}{c}6.00 \pm 0.89 \\
6.67 \pm 1.37 \\
1.83 \pm 0.41 \\
2.33 \pm 0.52 \\
100.0\end{array}$ & $\begin{array}{c}3.33 \pm 0.52 \\
5.33 \pm 1.37 \\
0.83 \pm 0.41 \\
1.33 \pm 1.03 \\
66.7\end{array}$ & $\begin{array}{c}3.17 \pm 0.41 \\
5.17 \pm 1.47 \\
0.83 \pm 0.41 \\
1.00 \pm 1.09 \\
50.0\end{array}$ \\
\hline 10 & $\begin{array}{c}\text { PD }(\mathrm{mm}) \\
\text { CAL }(\mathrm{mm}) \\
\text { PI } \\
\text { GI } \\
\text { BOP }(\%)\end{array}$ & $\begin{array}{c}5.40 \pm 0.55 \\
5.40 \pm 0.55 \\
0.80 \pm 1.09 \\
1.00 \pm 0.00 \\
0.0\end{array}$ & $\begin{array}{c}3.80 \pm 0.45 \\
3.80 \pm 0.45 \\
0.20 \pm 0.45 \\
0.80 \pm 0.84 \\
20.0\end{array}$ & $\begin{array}{c}3.80 \pm 0.45 \\
3.80 \pm 0.45 \\
0.40 \pm 0.55 \\
1.20 \pm 1.09 \\
60.0\end{array}$ & $\begin{array}{c}5.57 \pm 0.79 \\
5.57 \pm 0.79 \\
1.43 \pm 0.79 \\
1.43 \pm 0.54 \\
42.9\end{array}$ & $\begin{array}{c}4.29 \pm 0.95 \\
4.71 \pm 1.38 \\
0.71 \pm 0.49 \\
1.29 \pm 0.95 \\
57.1\end{array}$ & $\begin{array}{c}3.57 \pm 0.79 \\
4.00 \pm 1.00 \\
0.71 \pm 0.49 \\
1.29 \pm 0.76 \\
42.9\end{array}$ \\
\hline 11 & $\begin{array}{c}\text { PD }(\mathrm{mm}) \\
\text { CAL }(\mathrm{mm}) \\
\text { PI } \\
\text { GI } \\
\text { BOP }(\%)\end{array}$ & $\begin{array}{c}5.00 \pm 0.00 \\
6.50 \pm 0.71 \\
1.50 \pm 0.71 \\
1.00 \pm 0.00 \\
0.0\end{array}$ & $\begin{array}{c}3.50 \pm 0.71 \\
6.00 \pm 2.83 \\
0.50 \pm 0.71 \\
2.00 \pm 0.00 \\
100.0\end{array}$ & $\begin{array}{c}3.50 \pm 0.71 \\
6.00 \pm 2.83 \\
0.50 \pm 0.71 \\
0.00 \pm 0.00 \\
0.0\end{array}$ & $\begin{array}{c}5.00 \pm 0.00 \\
5.00 \pm 0.00 \\
2.00 \pm 0.00 \\
1.67 \pm 0.58 \\
66.7\end{array}$ & $\begin{array}{c}3.33 \pm 0.58 \\
3.67 \pm 1.16 \\
0.00 \pm 0.00 \\
2.00 \pm 0.00 \\
100.0\end{array}$ & $\begin{array}{c}3.33 \pm 0.58 \\
3.67 \pm 1.16 \\
0.00 \pm 0.00 \\
0.00 \pm 0.00 \\
0.0\end{array}$ \\
\hline 12 & $\begin{array}{c}\text { PD }(\mathrm{mm}) \\
\text { CAL (mm) } \\
\text { PI } \\
\text { GI } \\
\text { BOP }(\%)\end{array}$ & $\begin{array}{c}5.75 \pm 0.50 \\
5.75 \pm 0.50 \\
2.00 \pm 0.00 \\
1.75 \pm 0.50 \\
75.0\end{array}$ & $\begin{array}{c}4.75 \pm 0.50 \\
4.75 \pm 0.50 \\
0.00 \pm 0.00 \\
0.50 \pm 1.00 \\
25.0\end{array}$ & $\begin{array}{c}3.75 \pm 0.96 \\
3.75 \pm 0.96 \\
0.00 \pm 0.00 \\
0.25 \pm 0.50 \\
0.0\end{array}$ & $\begin{array}{c}5.00 \pm 0.00 \\
5.00 \pm 0.00 \\
2.00 \pm 0.00 \\
2.00 \pm 0.00 \\
100.0\end{array}$ & $\begin{array}{c}3.67 \pm 0.58 \\
3.67 \pm 0.58 \\
0.67 \pm 0.58 \\
1.00 \pm 0.00 \\
33.3\end{array}$ & $\begin{array}{c}3.33 \pm 0.58 \\
3.33 \pm 0.58 \\
0.00 \pm 0.00 \\
0.33 \pm 0.58 \\
0.0\end{array}$ \\
\hline 13 & $\begin{array}{c}\text { PD (mm) } \\
\text { CAL (mm) } \\
\text { PI } \\
\text { GI } \\
\text { BOP }(\%)\end{array}$ & $\begin{array}{c}5.44 \pm 0.53 \\
6.67 \pm 2.06 \\
1.67 \pm 0.71 \\
1.56 \pm 0.53 \\
55.6\end{array}$ & $\begin{array}{c}4.44 \pm 1.24 \\
5.78 \pm 2.68 \\
1.67 \pm 0.50 \\
1.33 \pm 1.00 \\
66.7\end{array}$ & $\begin{array}{c}4.22 \pm 1.20 \\
5.56 \pm 2.65 \\
0.89 \pm 0.78 \\
1.22 \pm 0.97 \\
55.6\end{array}$ & $\begin{array}{c}5.00 \pm 0.00 \\
6.25 \pm 0.96 \\
1.50 \pm 1.00 \\
1.50 \pm 0.58 \\
50.0\end{array}$ & $\begin{array}{c}4.75 \pm 0.50 \\
6.00 \pm 1.41 \\
1.25 \pm 0.50 \\
2.00 \pm 0.00 \\
100.0\end{array}$ & $\begin{array}{c}4.50 \pm 0.58 \\
6.00 \pm 1.83 \\
1.00 \pm 0.82 \\
1.00 \pm 1.16 \\
50.0\end{array}$ \\
\hline 14 & $\begin{array}{c}\text { PD }(\mathrm{mm}) \\
\text { CAL }(\mathrm{mm}) \\
\text { PI } \\
\text { GI } \\
\text { BOP }(\%)\end{array}$ & $\begin{array}{c}5.71 \pm 0.95 \\
5.57 \pm 0.98 \\
1.14 \pm 0.69 \\
2.00 \pm 0.00 \\
100.0\end{array}$ & $\begin{array}{c}3.71 \pm 0.76 \\
3.43 \pm 0.54 \\
1.14 \pm 0.38 \\
0.71 \pm 0.95 \\
28.6\end{array}$ & $\begin{array}{c}3.43 \pm 0.54 \\
3.29 \pm 0.49 \\
0.14 \pm 0.38 \\
0.43 \pm 0.79 \\
14.3\end{array}$ & $\begin{array}{c}5.92 \pm 0.95 \\
5.92 \pm 0.95 \\
1.77 \pm 0.44 \\
2.00 \pm 0.00 \\
100.0\end{array}$ & $\begin{array}{c}3.92 \pm 0.76 \\
4.15 \pm 0.69 \\
1.15 \pm 0.99 \\
1.00 \pm 0.91 \\
38.5\end{array}$ & $\begin{array}{c}3.77 \pm 0.73 \\
4.15 \pm 0.80 \\
0.92 \pm 0.86 \\
1.23 \pm 0.93 \\
53.8\end{array}$ \\
\hline 15 & $\begin{array}{c}\text { PD }(\mathrm{mm}) \\
\text { CAL }(\mathrm{mm}) \\
\text { PI } \\
\text { GI } \\
\text { BOP }(\%)\end{array}$ & $\begin{array}{c}5.20 \pm 0.45 \\
5.60 \pm 0.89 \\
1.80 \pm 0.45 \\
1.60 \pm 0.55 \\
60.0\end{array}$ & $\begin{array}{c}3.20 \pm 0.45 \\
3.60 \pm 0.89 \\
1.40 \pm 0.55 \\
1.60 \pm 0.89 \\
80.0\end{array}$ & $\begin{array}{c}3.00 \pm 0.00 \\
3.40 \pm 0.89 \\
1.20 \pm 0.84 \\
1.20 \pm 0.84 \\
40.0\end{array}$ & $\begin{array}{c}5.00 \pm 0.00 \\
5.33 \pm 0.58 \\
2.33 \pm 0.58 \\
1.33 \pm 0.58 \\
33.3\end{array}$ & $\begin{array}{c}3.67 \pm 0.58 \\
4.67 \pm 1.16 \\
0.33 \pm 0.58 \\
1.00 \pm 1.00 \\
33.3\end{array}$ & $\begin{array}{c}3.00 \pm 0.00 \\
4.33 \pm 1.16 \\
0.33 \pm 0.58 \\
1.33 \pm 1.16 \\
66.7\end{array}$ \\
\hline 16 & $\begin{array}{c}\text { PD }(\mathrm{mm}) \\
\text { CAL }(\mathrm{mm}) \\
\text { PI } \\
\text { GI } \\
\text { BOP }(\%)\end{array}$ & $\begin{array}{c}5.38 \pm 0.52 \\
5.75 \pm 0.71 \\
1.00 \pm 0.93 \\
1.88 \pm 0.64 \\
75.0\end{array}$ & $\begin{array}{c}4.13 \pm 0.64 \\
4.50 \pm 0.54 \\
0.63 \pm 0.52 \\
1.00 \pm 1.06 \\
50.0\end{array}$ & $\begin{array}{c}4.00 \pm 0.54 \\
4.38 \pm 0.52 \\
0.63 \pm 0.52 \\
0.50 \pm 0.54 \\
0.0\end{array}$ & $\begin{array}{c}5.00 \pm 0.00 \\
5.00 \pm 0.00 \\
1.00 \pm 1.00 \\
/ 2.00 \pm 0.00 \\
100.0\end{array}$ & $\begin{array}{c}3.67 \pm 0.58 \\
3.67 \pm 0.58 \\
0.33 \pm 0.58 \\
0.33 \pm 0.58 \\
0.0\end{array}$ & $\begin{array}{c}3.67 \pm 0.58 \\
3.67 \pm 0.58 \\
0.33 \pm 0.58 \\
0.33 \pm 0.58 \\
0.0\end{array}$ \\
\hline 17 & $\begin{array}{c}\text { PD }(\mathrm{mm}) \\
\text { CAL }(\mathrm{mm}) \\
\text { PI } \\
\text { GI } \\
\text { BOP }(\%)\end{array}$ & $\begin{array}{c}5.53 \pm 0.62 \\
5.65 \pm 0.61 \\
1.59 \pm 0.51 \\
1.76 \pm 0.44 \\
76.5\end{array}$ & $\begin{array}{c}3.88 \pm 0.60 \\
4.00 \pm 0.71 \\
0.35 \pm 0.49 \\
1.41 \pm 0.87 \\
64.7\end{array}$ & $\begin{array}{c}3.53 \pm 0.51 \\
3.71 \pm 0.59 \\
0.24 \pm 0.44 \\
0.88 \pm 0.78 \\
23.5\end{array}$ & $\begin{array}{c}5.42 \pm 0.52 \\
5.50 \pm 0.52 \\
1.67 \pm 0.65 \\
1.92 \pm 0.29 \\
91.7\end{array}$ & $\begin{array}{c}3.92 \pm 0.52 \\
4.08 \pm 0.29 \\
0.67 \pm 0.49 \\
0.92 \pm 0.90 \\
33.3\end{array}$ & $\begin{array}{c}3.92 \pm 0.52 \\
4.08 \pm 0.29 \\
0.50 \pm 0.52 \\
0.67 \pm 0.65 \\
8.3\end{array}$ \\
\hline 18 & $\begin{array}{c}\text { PD }(\mathrm{mm}) \\
\text { CAL (mm) } \\
\text { PI } \\
\text { GI } \\
\text { BOP }(\%)\end{array}$ & $\begin{array}{c}5.33 \pm 0.49 \\
5.17 \pm 0.58 \\
1.33 \pm 0.78 \\
1.83 \pm 0.39 \\
83.3\end{array}$ & $\begin{array}{c}3.67 \pm 0.78 \\
3.67 \pm 1.30 \\
0.75 \pm 0.45 \\
1.00 \pm 1.04 \\
50.0\end{array}$ & $\begin{array}{c}3.58 \pm 0.79 \\
3.67 \pm 1.30 \\
0.00 \pm 0.00 \\
1.33 \pm 0.99 \\
66.7\end{array}$ & $\begin{array}{c}5.43 \pm 0.54 \\
5.43 \pm 0.54 \\
1.57 \pm 0.54 \\
2.00 \pm 0.00 \\
100.0\end{array}$ & $\begin{array}{c}3.43 \pm 0.79 \\
3.43 \pm 0.79 \\
0.57 \pm 0.54 \\
1.71 \pm 0.76 \\
85.7\end{array}$ & $\begin{array}{c}3.43 \pm 0.79 \\
3.57 \pm 0.98 \\
0.14 \pm 0.38 \\
1.00 \pm 1.00 \\
42.9\end{array}$ \\
\hline 19 & $\begin{array}{c}\mathrm{PD}(\mathrm{mm}) \\
\text { CAL }(\mathrm{mm}) \\
\text { PI } \\
\text { GI } \\
\text { BOP }(\%)\end{array}$ & $\begin{array}{c}5.56 \pm 0.88 \\
6.44 \pm 1.42 \\
2.00 \pm 0.00 \\
2.44 \pm 0.53 \\
100.0\end{array}$ & $\begin{array}{c}4.44 \pm 0.88 \\
5.33 \pm 1.12 \\
2.44 \pm 0.53 \\
2.00 \pm 0.00 \\
100.0\end{array}$ & $\begin{array}{c}4.33 \pm 0.71 \\
5.78 \pm 1.20 \\
1.78 \pm 0.67 \\
2.00 \pm 0.00 \\
100.0\end{array}$ & $\begin{array}{c}5.69 \pm 1.11 \\
6.85 \pm 1.07 \\
2.38 \pm 0.65 \\
2.69 \pm 0.48 \\
100.0\end{array}$ & $\begin{array}{c}4.00 \pm 0.58 \\
5.92 \pm 0.86 \\
2.00 \pm 0.00 \\
1.77 \pm 0.44 \\
76.9\end{array}$ & $\begin{array}{c}3.77 \pm 0.44 \\
6.00 \pm 0.91 \\
1.69 \pm 0.48 \\
1.69 \pm 0.48 \\
69.2\end{array}$ \\
\hline
\end{tabular}


Mean patient clinical parameters (Cont.)

\begin{tabular}{|c|c|c|c|c|c|c|c|}
\hline \multirow[t]{2}{*}{$\begin{array}{c}\text { Particip } \\
\text { ant }\end{array}$} & \multirow{2}{*}{ Parameters } & \multicolumn{3}{|c|}{$\begin{array}{c}\mathrm{SRP}+\mathrm{PB} \\
(\mathrm{n}=271)\end{array}$} & \multicolumn{3}{|c|}{$\begin{array}{c}\mathrm{SRP}+\mathrm{AP} \\
(\mathrm{n}=292)\end{array}$} \\
\hline & & Baseline & 3 months & 6 months & Baseline & 3 months & 6 months \\
\hline 20 & $\begin{array}{c}\text { PD }(\mathrm{mm}) \\
\text { CAL (mm) } \\
\text { PI } \\
\text { GI } \\
\text { BOP }(\%)\end{array}$ & $\begin{array}{c}6.75 \pm 1.49 \\
7.50 \pm 1.93 \\
1.75 \pm 0.46 \\
2.75 \pm 0.71 \\
87.5\end{array}$ & $\begin{array}{c}6.25 \pm 1.83 \\
7.25 \pm 2.32 \\
0.75 \pm 0.46 \\
0.75 \pm 0.46 \\
0.0\end{array}$ & $\begin{array}{c}5.88 \pm 1.64 \\
7.00 \pm 2.00 \\
1.38 \pm 0.92 \\
1.25 \pm 0.46 \\
25.0\end{array}$ & $\begin{array}{c}5.91 \pm 1.22 \\
5.82 \pm 1.33 \\
2.00 \pm 0.00 \\
/ 2.82 \pm 0.41 \\
100.0\end{array}$ & $\begin{array}{c}4.91 \pm 1.70 \\
5.09 \pm 1.58 \\
0.45 \pm 0.52 \\
1.55 \pm 0.52 \\
54.5\end{array}$ & $\begin{array}{c}4.09 \pm 1.04 \\
4.27 \pm 0.91 \\
1.00 \pm 1.00 \\
1.45 \pm 0.52 \\
45.5\end{array}$ \\
\hline 21 & $\begin{array}{c}\text { PD (mm) } \\
\text { CAL (mm) } \\
\text { PI } \\
\text { GI } \\
\text { BOP }(\%)\end{array}$ & $\begin{array}{c}6.50 \pm 0.84 \\
6.50 \pm 1.23 \\
2.00 \pm 0.00 \\
2.00 \pm 0.00 \\
100.0\end{array}$ & $\begin{array}{c}4.00 \pm 0.89 \\
4.67 \pm 1.37 \\
0.17 \pm 0.41 \\
1.83 \pm 0.41 \\
83.3\end{array}$ & $\begin{array}{c}3.67 \pm 0.52 \\
4.50 \pm 1.23 \\
0.50 \pm 0.84 \\
1.67 \pm 082 \\
83.3\end{array}$ & $\begin{array}{c}6.50 \pm 0.71 \\
6.00 \pm 1.41 \\
2.00 \pm 0.00 \\
/ 2.00 \pm 0.00 \\
100.0\end{array}$ & $\begin{array}{c}3.00 \pm 0.00 \\
5.50 \pm 0.71 \\
1.00 \pm 0.00 \\
2.00 \pm 0.00 \\
100.0\end{array}$ & $\begin{array}{c}3.00 \pm 0.00 \\
5.50 \pm 0.71 \\
0.00 \pm 0.00 \\
2.00 \pm 0.00 \\
100.0\end{array}$ \\
\hline 22 & $\begin{array}{c}\text { PD (mm) } \\
\text { CAL (mm) } \\
\text { PI } \\
\text { GI } \\
\text { BOP }(\%)\end{array}$ & $\begin{array}{c}5.75 \pm 1.04 \\
5.88 \pm 0.99 \\
1.00 \pm 0.93 \\
2.13 \pm 0.64 \\
87.5\end{array}$ & $\begin{array}{c}3.63 \pm 0.74 \\
4.00 \pm 0.76 \\
1.38 \pm 0.74 \\
0.75 \pm 0.89 \\
25.0\end{array}$ & $\begin{array}{c}3.50 \pm 0.76 \\
3.88 \pm 0.84 \\
0.75 \pm 0.71 \\
0.75 \pm 1.04 \\
37.5\end{array}$ & $\begin{array}{c}5.83 \pm 1.27 \\
5.92 \pm 1.51 \\
1.42 \pm 0.79 \\
2.58 \pm 0.67 \\
91.7\end{array}$ & $\begin{array}{c}3.08 \pm 0.79 \\
3.67 \pm 1.07 \\
0.58 \pm 0.79 \\
1.25 \pm 0.87 \\
50.0\end{array}$ & $\begin{array}{c}3.08 \pm 0.79 \\
3.75 \pm 1.14 \\
1.75 \pm 0.45 \\
1.00 \pm 0.74 \\
25.0\end{array}$ \\
\hline 23 & $\begin{array}{c}\text { PD }(\mathrm{mm}) \\
\text { CAL (mm) } \\
\text { PI } \\
\text { GI } \\
\text { BOP }(\%) \\
\end{array}$ & $\begin{array}{c}5.56 \pm 0.53 \\
5.56 \pm 0.53 \\
0.11 \pm 0.33 \\
1.67 \pm .071 \\
44.4\end{array}$ & $\begin{array}{c}4.44 \pm 0.53 \\
4.44 \pm 0.53 \\
0.00 \pm 0.00 \\
0.67 \pm 1.00 \\
33.3\end{array}$ & $\begin{array}{c}4.44 \pm 0.53 \\
4.67 \pm 0.87 \\
0.00 \pm 0.00 \\
0.44 \pm 0.73 \\
11.1\end{array}$ & $\begin{array}{c}5.80 \pm 0.45 \\
5.60 \pm 1.14 \\
0.40 \pm 0.55 \\
1.80 \pm 0.45 \\
80.0\end{array}$ & $\begin{array}{c}4.80 \pm 0.84 \\
5.00 \pm 0.71 \\
0.00 \pm 0.00 \\
0.80 \pm 1.09 \\
40.0\end{array}$ & $\begin{array}{c}4.60 \pm 0.89 \\
5.20 \pm 0.84 \\
0.20 \pm 0.45 \\
1.60 \pm 0.89 \\
80.0\end{array}$ \\
\hline 24 & $\begin{array}{c}\text { PD (mm) } \\
\text { CAL (mm) } \\
\text { PI } \\
\text { GI } \\
\text { BOP }(\%)\end{array}$ & $\begin{array}{c}6.20 \pm 1.06 \\
8.85 \pm 1.66 \\
1.90 \pm 0.45 \\
2.25 \pm 0.55 \\
95.0\end{array}$ & $\begin{array}{c}3.50 \pm 0.61 \\
7.05 \pm 1.70 \\
0.95 \pm 0.39 \\
1.45 \pm 0.89 \\
70.0\end{array}$ & $\begin{array}{c}3.15 \pm 0.59 \\
7.25 \pm 2.17 \\
0.05 \pm 0.22 \\
0.80 \pm 0.83 \\
25.0\end{array}$ & $\begin{array}{c}6.41 \pm 1.00 \\
7.47 \pm 1.59 \\
1.88 \pm 0.49 \\
2.06 \pm 0.24 \\
94.1\end{array}$ & $\begin{array}{c}4.12 \pm 1.17 \\
7.06 \pm 1.52 \\
0.82 \pm 0.39 \\
1.53 \pm 0.80 \\
70.6\end{array}$ & $\begin{array}{c}3.82 \pm 1.02 \\
7.06 \pm 1.64 \\
0.06 \pm 0.24 \\
1.53 \pm 0.72 \\
64.7\end{array}$ \\
\hline 25 & $\begin{array}{c}\text { PD (mm) } \\
\text { CAL (mm) } \\
\text { PI } \\
\text { GI } \\
\text { BOP }(\%) \\
\end{array}$ & $\begin{array}{c}6.17 \pm 0.75 \\
6.17 \pm 0.75 \\
1.67 \pm 0.52 \\
2.33 \pm 0.52 \\
100.0\end{array}$ & $\begin{array}{c}3.50 \pm 0.55 \\
3.50 \pm 0.55 \\
0.67 \pm 0.82 \\
0.50 \pm 0.84 \\
16.7\end{array}$ & $\begin{array}{c}3.50 \pm 0.55 \\
3.50 \pm 0.55 \\
0.50 \pm 0.55 \\
1.00 \pm 1.09 \\
50.0\end{array}$ & $\begin{array}{c}5.55 \pm 0.52 \\
5.45 \pm 0.52 \\
1.64 \pm 0.51 \\
1.82 \pm 0.41 \\
81.8 \\
\end{array}$ & $\begin{array}{c}3.18 \pm 0.41 \\
3.18 \pm 0.41 \\
1.09 \pm 0.94 \\
1.09 \pm 0.83 \\
36.4\end{array}$ & $\begin{array}{c}3.55 \pm 0.69 \\
3.55 \pm 0.69 \\
0.73 \pm 0.47 \\
0.91 \pm 1.04 \\
45.5\end{array}$ \\
\hline 26 & $\begin{array}{c}\text { PD (mm) } \\
\text { CAL (mm) } \\
\text { PI } \\
\text { GI } \\
\text { BOP }(\%)\end{array}$ & $\begin{array}{c}7.14 \pm 1.35 \\
5.86 \pm 1.35 \\
1.57 \pm 0.79 \\
2.57 \pm 0.54 \\
85.7\end{array}$ & $\begin{array}{c}4.29 \pm 1.11 \\
3.86 \pm 1.07 \\
0.43 \pm 0.79 \\
1.43 \pm 0.54 \\
42.9\end{array}$ & $\begin{array}{c}4.29 \pm 1.11 \\
4.14 \pm 1.35 \\
0.71 \pm 0.95 \\
0.71 \pm 0.95 \\
28,6\end{array}$ & $\begin{array}{c}7.38 \pm 0.74 \\
6.63 \pm 0.52 \\
1.38 \pm 0.74 \\
2.88 \pm 0.35 \\
87.5\end{array}$ & $\begin{array}{c}5.00 \pm 1.07 \\
4.75 \pm 0.71 \\
0.75 \pm 1.04 \\
1.63 \pm 0.74 \\
75.0\end{array}$ & $\begin{array}{c}4.25 \pm 0.89 \\
4.50 \pm 0.76 \\
0.50 \pm 0.54 \\
1.50 \pm 0.93 \\
75.0\end{array}$ \\
\hline 27 & $\begin{array}{c}\mathrm{PD}(\mathrm{mm}) \\
\text { CAL }(\mathrm{mm}) \\
\text { PI } \\
\text { GI } \\
\text { BOP }(\%) \\
\end{array}$ & $\begin{array}{c}5.00 \pm 0.00 \\
3.50 \pm 0.58 \\
2.00 \pm 0.00 \\
2.75 \pm 0.50 \\
100.0\end{array}$ & $\begin{array}{c}3.75 \pm 0.50 \\
2.25 \pm 0.50 \\
1.00 \pm 0.00 \\
0.00 \pm 0.00 \\
0.0\end{array}$ & $\begin{array}{c}3.75 \pm 0.50 \\
3.25 \pm 0.96 \\
1.50 \pm 1.00 \\
1.50 \pm 0.58 \\
50.0\end{array}$ & $\begin{array}{c}5.75 \pm 1.17 \\
5.38 \pm 0.92 \\
2.00 \pm 0.00 \\
2.00 \pm 0.00 \\
100.0\end{array}$ & $\begin{array}{c}4.38 \pm 0.74 \\
4.00 \pm 0.93 \\
0.50 \pm 0.76 \\
1.25 \pm 1.04 \\
62.5 \\
\end{array}$ & $\begin{array}{c}4.00 \pm 0.54 \\
3.75 \pm 0.46 \\
0.50 \pm 0.76 \\
1.50 \pm 0.54 \\
50.0\end{array}$ \\
\hline 28 & $\begin{array}{c}\mathrm{PD}(\mathrm{mm}) \\
\text { CAL }(\mathrm{mm}) \\
\text { PI } \\
\text { GI } \\
\text { BOP }(\%) \\
\end{array}$ & $\begin{array}{c}5.63 \pm 1.06 \\
5.75 \pm 1.39 \\
1.75 \pm 0.71 \\
2.00 \pm 0.00 \\
100.0\end{array}$ & $\begin{array}{c}4.63 \pm 1.41 \\
5.00 \pm 1.85 \\
1.00 \pm 0.00 \\
1.38 \pm 0.92 \\
62.5 \\
\end{array}$ & $\begin{array}{c}4.63 \pm 1.41 \\
5.13 \pm 1.96 \\
0.38 \pm 0.52 \\
1.75 \pm 0.71 \\
87.5 \\
\end{array}$ & $\begin{array}{c}6.00 \pm 1.27 \\
6.00 \pm 1.27 \\
1.83 \pm 0.41 \\
2.00 \pm 0.00 \\
100.0 \\
\end{array}$ & $\begin{array}{c}4.50 \pm 0.84 \\
4.83 \pm 0.98 \\
1.50 \pm 0.55 \\
1.17 \pm 0.98 \\
50.0\end{array}$ & $\begin{array}{c}4.50 \pm 0.84 \\
5.33 \pm 1.37 \\
0.83 \pm 0.98 \\
0.67 \pm 1.03 \\
33.3 \\
\end{array}$ \\
\hline 29 & $\begin{array}{c}\mathrm{PD}(\mathrm{mm}) \\
\text { CAL }(\mathrm{mm}) \\
\text { PI } \\
\text { GI } \\
\text { BOP }(\%)\end{array}$ & $\begin{array}{c}5.29 \pm 0.49 \\
7.71 \pm 1.60 \\
2.00 \pm 0.00 \\
2.71 \pm 0.76 \\
14.3\end{array}$ & $\begin{array}{c}4.57 \pm 0.54 \\
7.29 \pm 1.60 \\
0.71 \pm 0.49 \\
1.00 \pm 0.82 \\
28.6\end{array}$ & $\begin{array}{c}4.43 \pm 0.54 \\
7.14 \pm 1.57 \\
1.86 \pm 0.38 \\
1.43 \pm 0.79 \\
57.1\end{array}$ & $\begin{array}{c}5.50 \pm 1.23 \\
7.17 \pm 2.14 \\
1.83 \pm 0.41 \\
1.33 \pm 0.52 \\
33.3\end{array}$ & $\begin{array}{c}4.33 \pm 0.82 \\
6.33 \pm 1.63 \\
0.33 \pm 0.52 \\
1.33 \pm 0.82 \\
50.0\end{array}$ & $\begin{array}{c}4.17 \pm 0.75 \\
6.17 \pm 1.47 \\
1.50 \pm 0.55 \\
2.00 \pm 0.00 \\
100.0\end{array}$ \\
\hline 30 & $\begin{array}{c}\text { PD }(\mathrm{mm}) \\
\text { CAL }(\mathrm{mm}) \\
\text { PI } \\
\text { GI } \\
\text { BOP }(\%)\end{array}$ & $\begin{array}{c}7.20 \pm 1.55 \\
8.20 \pm 1.99 \\
1.30 \pm 0.82 \\
1.90 \pm 0.32 \\
90.0\end{array}$ & $\begin{array}{c}5.10 \pm 1.66 \\
6.60 \pm 2.12 \\
0.60 \pm 0.52 \\
1.10 \pm 0.88 \\
40.0\end{array}$ & $\begin{array}{c}4.50 \pm 1.51 \\
6.00 \pm 2.00 \\
0.60 \pm 0.84 \\
1.10 \pm 0.99 \\
50.0\end{array}$ & $\begin{array}{c}5.00 \pm 0.00 \\
5.50 \pm 0.71 \\
2.00 \pm 0.00 \\
2.00 \pm 0.00 \\
100.0\end{array}$ & $\begin{array}{c}3.00 \pm 0.00 \\
4.50 \pm 0.71 \\
1.00 \pm 0.00 \\
0.00 \pm 0.00 \\
0.0\end{array}$ & $\begin{array}{c}3.00 \pm 0.00 \\
5.00 \pm 1.41 \\
2.00 \pm 0.00 \\
0.00 \pm 0.00 \\
0.0\end{array}$ \\
\hline
\end{tabular}


Mean patient clinical parameters (Cont.)

\begin{tabular}{|c|c|c|c|c|c|c|c|}
\hline \multirow{2}{*}{$\begin{array}{c}\text { Particip } \\
\text { ant }\end{array}$} & \multirow[t]{2}{*}{ Parameters } & \multicolumn{3}{|c|}{$\begin{array}{c}S R P+P B \\
(n=271)\end{array}$} & \multicolumn{3}{|c|}{$\begin{array}{c}\text { SRP+AP } \\
(n=292)\end{array}$} \\
\hline & & Baseline & 3 months & 6 months & Baseline & 3 months & 6 months \\
\hline 31 & $\begin{array}{c}\text { PD }(\mathrm{mm}) \\
\text { CAL }(\mathrm{mm}) \\
\text { PI } \\
\text { GI } \\
\text { BOP }(\%)\end{array}$ & $\begin{array}{c}7.07 \pm 1.58 \\
8.40 \pm 1.18 \\
2.00 \pm 0.00 \\
2.00 \pm 0.39 \\
93.3\end{array}$ & $\begin{array}{c}4.13 \pm 1.55 \\
6.93 \pm 1.67 \\
1.20 \pm 0.68 \\
1.60 \pm 0.63 \\
66.7 \\
\end{array}$ & $\begin{array}{c}3.87 \pm 1.41 \\
7.13 \pm 1.55 \\
0.13 \pm 0.35 \\
0.87 \pm 0.92 \\
50.0\end{array}$ & $\begin{array}{c}6.78 \pm 1.68 \\
7.61 \pm 1.69 \\
2.00 \pm 0.00 \\
2.26 \pm 0.45 \\
100.0\end{array}$ & $\begin{array}{c}3.43 \pm 0.59 \\
6.00 \pm 1.28 \\
1.26 \pm 0.69 \\
1.00 \pm 0.79 \\
0.0\end{array}$ & $\begin{array}{c}3.39 \pm 0.58 \\
6.30 \pm 1.36 \\
0.57 \pm 0.51 \\
1.48 \pm 0.73 \\
0.0\end{array}$ \\
\hline 32 & $\begin{array}{c}\text { PD }(\mathrm{mm}) \\
\text { CAL }(\mathrm{mm}) \\
\text { PI } \\
\text { GI } \\
\text { BOP }(\%) \\
\end{array}$ & $\begin{array}{c}5.50 \pm 0.79 \\
6.42 \pm 1.44 \\
2.00 \pm 0.00 \\
2.42 \pm 0.52 \\
100.0\end{array}$ & $\begin{array}{c}4.08 \pm 1.08 \\
5.17 \pm 1.69 \\
1.17 \pm 0.94 \\
1.50 \pm 0.91 \\
75.0\end{array}$ & $\begin{array}{c}3.92 \pm 1.08 \\
5.00 \pm 1.71 \\
0.83 \pm 0.94 \\
1.58 \pm 0.79 \\
75.0\end{array}$ & $\begin{array}{c}6.77 \pm 1.73 \\
8.00 \pm 2.49 \\
1.77 \pm 0.65 \\
2.50 \pm 0.51 \\
92.3 \\
\end{array}$ & $\begin{array}{c}4.38 \pm 1.20 \\
6.35 \pm 2.24 \\
1.08 \pm 0.69 \\
1.42 \pm 0.86 \\
65.4\end{array}$ & $\begin{array}{c}4.31 \pm 1.16 \\
6.65 \pm 2.08 \\
0.50 \pm 0.71 \\
1.00 \pm 0.89 \\
38.5\end{array}$ \\
\hline 33 & $\begin{array}{c}\text { PD }(\mathrm{mm}) \\
\text { CAL }(\mathrm{mm}) \\
\text { PI } \\
\text { GI } \\
\text { BOP }(\%) \\
\end{array}$ & $\begin{array}{c}5.80 \pm 0.63 \\
5.80 \pm 0.63 \\
1.60 \pm 0.52 \\
1.90 \pm 0.32 \\
90.0\end{array}$ & $\begin{array}{c}4.90 \pm 0.57 \\
5.50 \pm 0.71 \\
0.50 \pm 0.85 \\
1.40 \pm 0.52 \\
40.0\end{array}$ & $\begin{array}{c}4.10 \pm 0.74 \\
5.20 \pm 0.63 \\
0.50 \pm 0.53 \\
1.00 \pm 0.00 \\
0.0\end{array}$ & $\begin{array}{c}5.77 \pm 0.44 \\
5.77 \pm 0.44 \\
1.85 \pm 0.38 \\
1.85 \pm 0.38 \\
84.6\end{array}$ & $\begin{array}{c}3.38 \pm 0.51 \\
4.23 \pm 0.44 \\
0.54 \pm 0.52 \\
1.00 \pm 0.00 \\
0.0\end{array}$ & $\begin{array}{c}2.62 \pm 0.65 \\
3.15 \pm 0.69 \\
0.54 \pm 0.52 \\
1.00 \pm 0.00 \\
0.0\end{array}$ \\
\hline 34 & $\begin{array}{c}\text { PD }(\mathrm{mm}) \\
\text { CAL }(\mathrm{mm}) \\
\text { PI } \\
\text { GI } \\
\text { BOP }(\%) \\
\end{array}$ & $\begin{array}{c}6.89 \pm 1.45 \\
6.89 \pm 1.45 \\
2.00 \pm 0.00 \\
1.89 \pm 0.33 \\
88.9\end{array}$ & $\begin{array}{c}5.00 \pm 1.23 \\
5.00 \pm 1.23 \\
0.00 \pm 0.00 \\
1.00 \pm 0.00 \\
0.0\end{array}$ & $\begin{array}{c}4.89 \pm 1.05 \\
5.00 \pm 1.23 \\
0.00 \pm 0.00 \\
1.00 \pm 0.00 \\
0.0\end{array}$ & $\begin{array}{c}6.00 \pm 1.58 \\
6.00 \pm 1.58 \\
1.78 \pm 0.44 \\
1.78 \pm 0.44 \\
77.8\end{array}$ & $\begin{array}{c}4.11 \pm 1.27 \\
4.22 \pm 1.20 \\
0.44 \pm 0.53 \\
1.00 \pm 0.0 \\
0.0\end{array}$ & $\begin{array}{c}4.11 \pm 1.27 \\
4.22 \pm 1.20 \\
0.00 \pm 0.00 \\
1.00 \pm 0.00 \\
0.0\end{array}$ \\
\hline 35 & $\begin{array}{c}\text { PD }(\mathrm{mm}) \\
\text { CAL (mm) } \\
\text { PI } \\
\text { GI } \\
\text { BOP }(\%)\end{array}$ & $\begin{array}{c}6.33 \pm 1.21 \\
6.50 \pm 1.64 \\
1.50 \pm 0.55 \\
1.67 \pm 0.52 \\
66.7\end{array}$ & $\begin{array}{c}4.33 \pm 1.21 \\
5.83 \pm 1.72 \\
1.00 \pm 0.00 \\
1.00 \pm 0.00 \\
0.0\end{array}$ & $\begin{array}{c}4.17 \pm 0.98 \\
5.67 \pm 1.51 \\
0.33 \pm 0.52 \\
1.00 \pm 0.00 \\
0.0\end{array}$ & $\begin{array}{c}6.14 \pm 0.69 \\
7.00 \pm 0.82 \\
1.57 \pm 0.54 \\
1.57 \pm 0.54 \\
57.1\end{array}$ & $\begin{array}{c}4.29 \pm 0.49 \\
5.71 \pm 0.49 \\
1.00 \pm 0.00 \\
1.00 \pm 0.00 \\
0.0\end{array}$ & $\begin{array}{c}3.86 \pm 0.90 \\
5.43 \pm 0.98 \\
1.00 \pm 0.00 \\
1.00 \pm 0.00 \\
0.0\end{array}$ \\
\hline
\end{tabular}

Table 2: Clinical parameters at baseline, 3 months, and 6 months

\begin{tabular}{|c|c|c|c|c|c|c|}
\hline \multirow{3}{*}{ Parameters } & \multicolumn{3}{|c|}{$\begin{array}{c}\text { SRP+PB } \\
(\mathrm{n}=263)\end{array}$} & \multicolumn{3}{c|}{$\begin{array}{c}\text { SRP+AP } \\
(\mathrm{n}=263)\end{array}$} \\
\cline { 2 - 7 } & Baseline & 3 months & 6 months & Baseline & 3 months & 6 months \\
\hline PD $(\mathrm{mm})$ & $5.87 \pm 1.10$ & $4.10 \pm 1.17 \dagger$ & $3.84 \pm 1.09 \dagger \#$ & $5.94 \pm 1.15$ & $3.94 \pm 0.99 \dagger$ & $3.72 \pm 0.88 \dagger \#$ \\
CAL $(\mathrm{mm})$ & $6.43 \pm 1.66$ & $5.12 \pm 1.86 \dagger$ & $5.03 \pm 1.88 \dagger \#$ & $6.26 \pm 1.46$ & $4.88 \pm 1.55 \dagger$ & $4.83 \pm 1.61 \dagger$ \\
PI & $1.64 \pm 0.68$ & $0.90 \pm 0.78 \dagger$ & $0.63 \pm 0.78 \dagger \#$ & $1.78 \pm 0.62 *$ & $0.95 \pm 0.77 \dagger$ & $0.70 \pm 0.76 \dagger \#$ \\
GI & $2.01 \pm 0.56$ & $1.21 \pm 0.87 \dagger$ & $1.03 \pm 0.86 \dagger \#$ & $2.05 \pm 0.58$ & $1.29 \pm 0.81 \dagger$ & $1.17 \pm 0.83 \dagger \#$ \\
BOP $(\%)$ & 82.1 & $50.2 \dagger$ & $38.8 \dagger \#$ & 84.8 & $51.3 \dagger$ & $44.1 \dagger$ \\
\hline
\end{tabular}

†significant within-group differences compared with baseline $(p<0.05)$

\#significant within-group differences compared with 3 months $(p<0.05)$

*significant between-groups differences $(p<0.05)$

\section{Within-Group Analysis}

The data obtained at the 3-month and 6month examinations are presented in Table 1. The within-group analyses revealed that both treatment modalities resulted in significant differences from baseline with regard to $\mathrm{PD}$ reduction, $\mathrm{CAL}$ gain, $\mathrm{PI}$ reduction, GI reduction, and BOP reduction at 3 and 6 months. However, no changes were observed with regard to tooth mobility. Nevertheless, mobility decreased $(\mathrm{SRP}+\mathrm{PB}=10.34 \%, \quad \mathrm{SRP}+\mathrm{AP}=14.29 \%)$, whereas mobility increased $(\mathrm{SRP}+\mathrm{PB}=6.89 \%, \quad \mathrm{SRP}+\mathrm{AP}=15.97 \% ; \quad$ data not shown). 


\section{Between-Group Analysis}

\section{PD Reduction}

Significant between-group differences were found in PD reduction from baseline to 3 months in the SRP+AP group; however, no significant differences were found from baseline to 6 months within either group (Table 3).

Table 3: Between-group analyses revealed PD reductions and CAL gains at 3 and 6 months compared with baseline (mean \pm SD)

\begin{tabular}{|l|c|c|c|c|}
\hline \multirow{2}{*}{ Parameters } & \multicolumn{2}{|c|}{$\begin{array}{c}\text { SRP+PB } \\
(\mathrm{n}=263)\end{array}$} & \multicolumn{2}{c|}{$\begin{array}{c}\text { SRP+AP } \\
(\mathrm{n}=263)\end{array}$} \\
\cline { 2 - 5 } & $\begin{array}{c}\text { Baseline to } \\
3 \text { months }\end{array}$ & $\begin{array}{c}\text { Baseline to } \\
6 \text { months }\end{array}$ & $\begin{array}{c}\text { Baseline to } \\
3 \text { months }\end{array}$ & $\begin{array}{c}\text { Baseline to } \\
6 \text { months }\end{array}$ \\
\hline PD reduction $(\mathrm{mm})$ & $1.78 \pm 1.05$ & $2.04 \pm 1.08$ & $2.00 \pm 1.16^{*}$ & $2.22 \pm 1.13$ \\
CAL gain $(\mathrm{mm})$ & $1.31 \pm 1.18$ & $1.40 \pm 1.23$ & $1.38 \pm 1.19$ & $1.44 \pm 1.22$ \\
\hline
\end{tabular}

*significant between-group differences $(p<0.05)$

When baseline PD was used to divide each treatment group into 5- to 6-mm and 7- to 12-mm subgroups, no significant betweengroup differences were found at any observed time (Table 4). The percentage of resolved sites (i.e., $\mathrm{PD}$ reduction $\geq 2 \mathrm{~mm}$ ) in $\mathrm{SRP}+\mathrm{AP}(76.43 \%)$ and in SRP+PB $(68.06 \%)$ was no significant differences (Table 5).

Table 4: Between-group (i.e., 5 to $6 \mathrm{~mm}$ vs. 7 to $12 \mathrm{~mm}$ ) analyses of PD reduction and CAL gain at 3 and 6 months compared with baseline (mean \pm SD)

\begin{tabular}{|c|c|c|c|c|c|}
\hline \multirow{2}{*}{$\begin{array}{c}\text { Baseline } \\
\text { PD } \\
(\mathrm{mm})\end{array}$} & \multirow{2}{*}{$\begin{array}{l}\text { Time after } \\
\text { baseline }\end{array}$} & \multicolumn{2}{|c|}{$\mathrm{SRP}+\mathrm{PB}$} & \multicolumn{2}{|c|}{$\mathrm{SRP}+\mathrm{AP}$} \\
\hline & & $\begin{array}{l}\text { PD reduction } \\
(\mathrm{mm})\end{array}$ & $\begin{array}{l}\text { CAL gain } \\
(\mathrm{mm})\end{array}$ & $\begin{array}{l}\text { PD reduction } \\
(\mathrm{mm})\end{array}$ & $\begin{array}{l}\text { CAL gain } \\
(\mathrm{mm})\end{array}$ \\
\hline $\begin{array}{c}5-6 \\
(n=406)\end{array}$ & $\begin{array}{l}3 \text { months } \\
6 \text { months }\end{array}$ & $\begin{array}{c}1.54 \pm 0.86 \\
(n=208) \\
1.76 \pm 0.88 \dagger\end{array}$ & $\begin{array}{c}1.18 \pm 1.11 \\
(n=208) \\
1.27 \pm 1.17 \dagger\end{array}$ & $\begin{array}{c}1.67 \pm 0.82 \\
(\mathrm{n}=198) \\
1.83 \pm 0.78 \dagger\end{array}$ & $\begin{array}{c}1.19 \pm 0.99 \\
(\mathrm{n}=198) \\
1.23 \pm 1.04\end{array}$ \\
\hline $\begin{array}{c}7-12 \\
(n=120)\end{array}$ & 3 months & $\begin{array}{c}2.69 \pm 1.20 \\
(n=55) \\
3.07 \pm 1.17 \dagger\end{array}$ & $\begin{array}{c}1.82 \pm 1.29 \\
(n=55) \\
1.91 \pm 1.34\end{array}$ & $\begin{array}{c}3.00 \pm 1.44 \\
(n=65) \\
3.40 \pm 1.24 \dagger\end{array}$ & $\begin{array}{c}1.95 \pm 1.50 \\
(\mathrm{n}=65) \\
2.06 \pm 1.49\end{array}$ \\
\hline
\end{tabular}

†significant within-group differences $(p<0.05)$ 
Table 5: The percentage of sites with PD reductions of $<2 \mathbf{m m}$ or $\geq 2 \mathrm{~mm}$

\begin{tabular}{|c|c|c|c|c|}
\hline \multirow{2}{*}{$\begin{array}{c}\mathrm{PD} \\
\text { reduction } \\
(\mathrm{mm})\end{array}$} & \multicolumn{2}{|c|}{$\begin{array}{l}\mathrm{SRP}+\mathrm{PB} \\
(\mathrm{n}=263)\end{array}$} & \multicolumn{2}{|c|}{$\begin{array}{l}\mathrm{SRP}+\mathrm{AP} \\
(\mathrm{n}=263)\end{array}$} \\
\hline & $\begin{array}{l}\text { Baseline to } \\
3 \text { months }\end{array}$ & $\begin{array}{c}\text { Baseline to } \\
6 \text { months }\end{array}$ & $\begin{array}{c}\text { Baseline } \\
3 \text { months } \\
\end{array}$ & $\begin{array}{c}\text { Baseline to } \\
6 \text { months } \\
\end{array}$ \\
\hline $\begin{array}{l}<2 \\
\geq 2\end{array}$ & $\begin{array}{l}42.21 \\
57.79\end{array}$ & $\begin{array}{l}31.94 \\
68.06\end{array}$ & $\begin{array}{l}33.08 \\
66.92\end{array}$ & $\begin{array}{l}23.57 \\
76.43\end{array}$ \\
\hline$p$-value & \multicolumn{2}{|c|}{0.03} & \multicolumn{2}{|c|}{0.03} \\
\hline $\begin{array}{l}{ }^{*} \text { Adjusted } \\
p \text {-value }\end{array}$ & \multicolumn{2}{|c|}{0.06} & \multicolumn{2}{|c|}{0.06} \\
\hline
\end{tabular}

*=adjusted $p$-value for multiple comparison test

\section{CAL gain}

No significant differences were found between the two groups at either 3 months or 6 months (Table 2). When the baseline PD was used to divide each treatment group into 5- to 6-mm and 7- to $12-\mathrm{mm}$ subgroups, no significant differences were found between the two treatment groups at any time (Table 3). At 6 months, 51.33\% of the sites in the SRP+AP group demonstrated an attachment increases of $\geq 2 \mathrm{~mm}$ compared with $46.77 \%$ of those in the SRP+PB group; however, there were no significant differences between $\mathrm{SRP}+\mathrm{AP}$ and SRP+PB (Table 6).

Table 6: The percentage of sites with CAL gain of $<2 \mathrm{~mm}$ or $\geq 2 \mathrm{~mm}$

\begin{tabular}{|c|c|c|c|c|}
\hline \multirow{2}{*}{$\begin{array}{c}\text { CAL gain } \\
(\mathrm{mm})\end{array}$} & \multicolumn{2}{|c|}{$\begin{array}{c}\text { SRP+PB } \\
(\mathrm{n}=263)\end{array}$} & \multicolumn{2}{c|}{$\begin{array}{c}\text { SRP+AP } \\
(\mathrm{n}=263)\end{array}$} \\
\cline { 2 - 5 } & $\begin{array}{c}\text { Baseline to } \\
3 \text { months }\end{array}$ & $\begin{array}{c}\text { Baseline to } \\
6 \text { months }\end{array}$ & $\begin{array}{c}\text { Baseline to } \\
\text { 3 months }\end{array}$ & $\begin{array}{c}\text { Baseline to } \\
6 \text { months }\end{array}$ \\
\hline$<2$ & 57.03 & 53.23 & 53.99 & 48.67 \\
$\geq 2$ & 42.97 & 46.77 & 46.01 & 51.33 \\
\hline \multirow{2}{*}{0 -value } & \multicolumn{2}{|c|}{0.48} & \multicolumn{2}{|c|}{0.29} \\
\hline *Adjusted \\
$p$-value
\end{tabular}

*=adjusted $p$-value for multiple comparison test 


\section{Radiographic Examination}
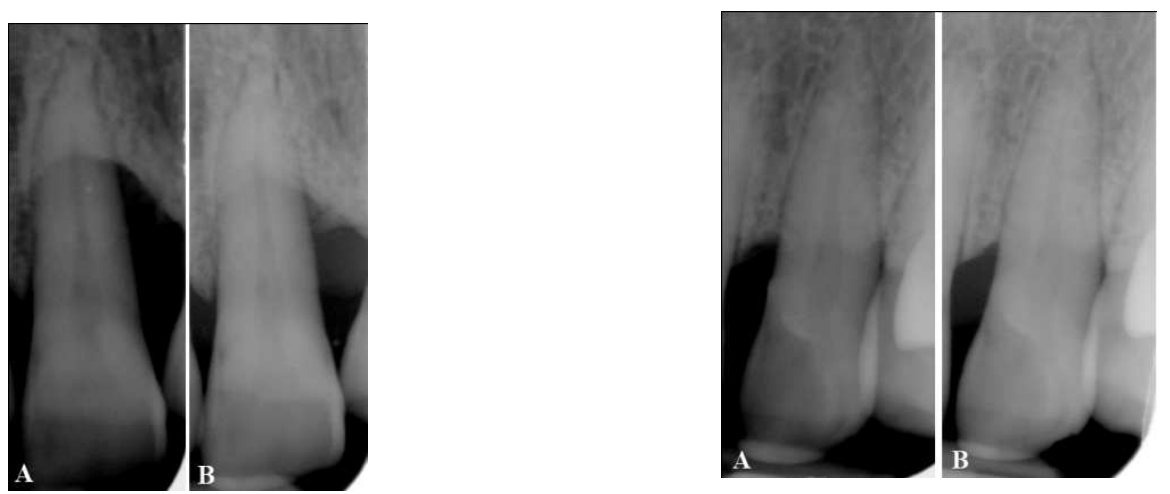

Figures 3 and 4 show radiographs at baseline and 6 months

Figure 3: Periapical radiographs taken at baseline (A) and 6 months (B) at the mesial aspect of Tooth 11 in the SRP+AP group

\section{Discussion}

This study evaluated the effectiveness of subgingivally delivered AP gel as an adjunct to treat patients with chronic periodontitis. This study used a splitmouth, single-blind, randomized controlled design to compare results between $\mathrm{SRP}+\mathrm{PB}$ and SRP+AP groups. The one operator collected data and the others who were blind to gel type administered PB or AP gels. Thus, examination bias did not occur. However, a double-blind design was not realistic for this study due to limitations regarding the color and bitter taste of the AP gel.

The split-mouth design allowed carryover effects to influence the treatment comparisons. The results of previous pharmacokinetic studies (Kuphasuk et al., 2004a, 2004b, 2008) suggest that these effects would not occur in the current study because andrographolide concentrations can be found in gingival crevicular fluid up to 24 hours after administration, and in saliva up to $1 / 2$ hour after administration; furthermore, the maximum concentration of andrographolide was less than the MIC $(537.70 \mu \mathrm{g} / \mathrm{ml})$. Examiner calibration was performed prior to the study, thereby resulting in strong reliability.
Figure 4: Periapical radiographs taken at baseline (A) and 6 months (B) at the mesial aspect of Tooth 22 in the SRP+PB group

A within-group analysis revealed that both treatment modalities improved clinical conditions with regard to $\mathrm{PD}$ reduction, CAL gain, PI reduction, GI reduction, and BOP reduction at 3 months and 6 months after baseline. This study resulted in PD reductions in the $\mathrm{SRP}+\mathrm{AP}$ group that were slightly less than those observed in a previous study (2.22 mm vs. $2.29 \mathrm{~mm}$; Boonchaipanichwatana, 2001), because PD reductions are greater for deeper PDs without surgery. Therefore, subgroups were created based on baseline PD by dividing each treatment group into 5- to 6$\mathrm{mm}$ and $7-$ to $12-\mathrm{mm}$ categories. Nevertheless, significant differences were not found between these subgroups at any observed time. This outcome might be because fewer sites existed within the 7- to 12-mm subgroup than in the 5- 6-mm subgroup with regard to baseline PD (55 vs. 208 in SRP+PB; 65 vs. 198 in SRP+AP).

The expected PD reductions and CAL gains after performing SRP in deep pockets were $2.16 \mathrm{~mm}$ and $1.19 \mathrm{~mm}$, respectively (Cobb, 1996). Between-group analyses did not demonstrate significant differences in PD reduction or $\mathrm{CAL}$ gain. At 6 months, $76.43 \%$ of the SRP+AP group demonstrated $\mathrm{PD}$ reduction $\geq 2 \mathrm{~mm}$ compared with $68.06 \%$ of those in the SRP+PB. In addition, $51.33 \%$ of the sites in 
the $\mathrm{SRP}+\mathrm{AP}$ group demonstrated CAL gain of $\geq 2 \mathrm{~mm}$ compared with $46.77 \%$ of sites in the SRP+PB group. However, the percentage of resolved sites (i.e. PD reduction $\geq 2 \mathrm{~mm}$, and attachment gain $\geq 2$ $\mathrm{mm}$ ) in the SRP+AP and in SRP+PB was no significant differences. Non-surgical treatments using AP gel as an adjunctive therapy tended to improve clinical reductions in PD and increases in CAL.

Both treatment groups showed improvements from baseline with regard to the PI, the GI, and BOP, and significant between-group differences were not observed. The GI lowered by one scoring level (i.e., the baseline score of 2.01 decreased to 1.03), which matches the results reported in an earlier study (Cobb, 2002). All three of these indices decreased after treatment in both groups; however, these differences were not significant. This finding might be due to the effectiveness of the regular oral hygiene that patients practiced to control supragingival plaque, which might also have resolved gingival inflammation and BOP. AP gel might not affect the PI, the GI, or BOP. These findings match those reported in previous studies (Axelsson \& Lindhe, 1978, Helldén et al., 1979).

Previous studies showed that AP gel is a useful adjunctive treatment in periodontal therapy (Rassameemasmaung et al., 1998, Atsawasuwan et al., 1998, Boonchaipanichwatana, 2001, Sirirat \& Rojanapanthu, 2003, Thawornrungroj, 2011). The effects of AP gel are due to its antibacterial activity (Amornchat et al., 1991), its anti-inflammatory action (Farnworth \& Bunyaprephatsara, 1992), and its biocompatibility with dentin surfaces, which enhances fibroblast attachment and migration (Hamasakwattanakul, 2004). The present study demonstrated that SRP+AP enhances $P D$ reduction and increases CAL, but these differences were not significant compared with the SRP+PB group. This result might be because SRP alone improves clinical parameters, and most of the selected sites were horizontal defects. Apart from this efficacy, importantly, the same local drugdelivery system might not work equally well in all sites or all patients due to significant variability (Kornman, 1993) in the composition of microorganisms, the effectiveness of AP gel against $P$. gingivalis and individual host response.

Decreased mobility is a desirable outcome after non-surgical periodontal therapy (Kerry et al., 1982). However, 27 of 235 teeth $(11.49 \%)$ showed deteriorated mobility. This outcome might be due to traumatic occlusion. Furthermore, heavy calculus deposits that splinted the teeth at baseline might have led to tooth mobility underestimation.

The results of the present single-center study, which represent a portion of a multicenter study, must be interpreted in the context of the limitations of the small number of selected sites with initial PDs of $\geq 7 \mathrm{~mm}$ or vertical defects. The improvements in PD and CAL might have been minimally influenced by the adjunctive use of AP gel due to improvements in the oral hygiene. Therefore, additional studies are needed with deep initial PDs and vertical defects according to radiographs.

\section{Conclusions}

The adjunctive use of $A P$ gel significantly reduced $P D$ and significantly improved attachment level from baseline. Additional studies are needed to assess this gel's performance in deep initial PDs with vertical defects according to radiographs.

\section{Acknowledgments}

A research grant from Mahidol University supported this research.

\section{References}

1. Adriaens, P.A., Edwards, C.A., De Boever, J.A. and Loesche, W.J. (1988) "Ultrastructural observations on bacterial invasion in cementum and radicular dentin of periodontally diseased human teeth," Journal of Periodontology, 59, 493-503.

2. Ainamo, J. and Bay, I. (1975) "Problems and proposals for recording gingivitis and 
plaque," International Dental Journal, 25, 229-235.

3. Amornchat, C., Kraivaphan, P., Kraivaphan, V. and Triratana, T. (1991) "The antibacterial activity of Andrographis paniculata crude extracts on oral bacteria," The Journal of the Dental Association of Thailand, 41, 178-185.

4. Anju, P., Sakena, R.P., Sakena, K.C., Srivastava, V. and Tendon, J.S. (1993) "Immunostimulant agent from Andrographis paniculata," Journal of Natural Products, 56, 995-999.

5. Atsawasuwan, P., Sirirat, M., Amornchat, C., Komwatchara, T., Rojanapanthu, P. and Yudhasaraprasithit, S. (1998) "Subgingival administration of Andrographis paniculata gel and Metronidazole gel as an adjunct in the treatment of adult periodontitis: Clinical and microbiological effects," Mahidol Journal, 5, 97-101.

6. Axelsson, P. and Lindhe, J. (1978) "Effect of controlled oral hygiene procedures on caries and periodontal disease in adults," Journal of Clinical Periodontology, 5 (2) 133-151.

7. Badersten, A., Nilveus, R. and Egelberg, J. (1990) "Scores of plaque, bleeding, suppuration and probing depth to predict probing attachment loss. 5 years of observation following nonsurgical periodontal therapy," Journal of Clinical Periodontology, 17, 102-107.

8. Boonchaipanichwatana, P. (2001) "The comparative clinical and microbiological effects of Andrographis paniculata gel and minocycline ointment as an adjunct in the treatment of early onset periodontitis" Thesis, Mahidol University.

9. Caffesse, R.G., Sweeney, P.L. and Smith, B.A. (1986) "Scaling and root planing with and without periodontal flap surgery," Journal of Clinical Periodontology, 13, 205210.

10.Cobb, C.M. (1996) "Non-surgical pocket therapy: mechanical," Annals of Periodontology, 1, 443-490.
11.Cobb, C.M. (2002) "Clinical significance of non-surgical periodontal therapy: an evidence-based perspective of scaling and root planning," Journal of Clinical Periodontology, 29 (Suppl. 2) 6-16.

12.Farnworth, N. and Bunyaprephatsara, N. (1992) "Thai medicinal plants recommended for primary health care system," Medicinal Plant information center, Faculty of Pharmacy, Mahidol University.

13.Haffajee, A.D. and Socransky, S.S. (1994) "Microbial etiological agents of destructive periodontal diseases," Periodontology 2000, 5, 78-111.

14.Hamasakwattanakul, A. (2004) "The effect of Andrographis paniculata gel on the migration of human periodontal ligament fibroblast," Thesis, Mahidol University.

15.Helldén, L.B., Listgarten, M.A. and Lindhe, J. (1979) "The effect of tetracycline and/or scaling on human periodontal disease," Journal of Clinical Periodontology, 6 (4) 222-230.

16.Hughes T.P. and Caffesse R.G. (1978) "Gingival changes following scaling, root planing and oral hygiene. A biometric evaluation," Journal of Periodontology, 49, 245-252.

17.Isidor, F. and Karring, T. (1986) "Longterm effect of surgical and non-surgical periodontal treatment. A 5-year clinical study," Journal of Periodontal Research, 21, 462-472.

18.Jewvachdamrongkul, Y., Chokechaijaroenporn, 0., Chavalittumrong, P. and Dechatiwongse, T. (1987) "Chemical quality evaluation of Fah Talai Jone," The Bulletin of the Department of Medical Sciences. 29, 231-237.

19.Kerry, G.J., Morrison, E.C., Ramfjord, S.P., Hill, R.W., Caffesse, R.G., Nissle, R.R and Appleberry, E.A. (1982) "Effect of periodontal treatment on tooth mobility," Journal of Periodontology, 53 (10) 635-638. 
20.Knowles, J., Burgett, F., Morrison, E., Nissle, R. and Ramfjord, S. (1980) "Comparison of results following three modalities of periodontal therapy related to tooth type and initial pocket depth," Journal of Clinical Periodontology, 7, 32-47.

21.Kornman, K.S. (1993) "Controlledrelease local delivery antimicrobials in periodontics: prospects for the future," Journal of Periodontology, 64, 782-791.

22.Kuphasuk, Y., Kuttinanon, P., Sirirat, M., Rojanapanthu, P. and Gritsanapan, W. (2004a) "Retention of Andrographis paniculata gel in periodontal pocket," Mahidol Dental Journal, 24, 81-90.

23.Kuphasuk, Y., Supaongprapa, T., Sirirat, M., Rojanapanthu, P. and Gritsanapan, W. (2004b) "Concentration of Andrographis paniculata gel in periodontal pocket," Mahidol Dental Journal, 24, 91-102.

24.Kuphasuk, Y., Srichati, A., Sirirat, M. and Kasetsuwan, J. (2008) "Pharmacokinetic profile of a locally administered Andrographis paniculata gel in crevicular fluid saliva and blood plasma," Thai Journal Periodontology, 1, 38-47.

25.Listgarten, M.A., Lindhe, J. and Hellden, L. (1978) "Effect of tetracycline and/or scaling on human periodontal disease. Clinical, microbiological, and histological observations," Journal of Clinical Periodontology, 5, 246-271.

26.Löe, H. and Silness, J., (1963) "Periodontal Disease in Pregnancy. I. Prevalence and Severity," Acta Odontologica Scandinavica, 21, 533-551.

27. Meenatchisundaram, S., Parameswari, G., Subbraj, T., Suganya, T. and Michael, A. (2009) "Medicinal and Pharmacological Activities of Andrographis paniculata Review," Ethnobotanical Leaflets, 13, 55-58.

28. Miller, S.C. (1943) Textbook of Periodontia (2nd ed.), The Blakiston Co., Philadelphia, USA. The Blakiston Co.

29.Pihlstrom, B.L., Ortiz-Campos, C. and McHugh, R.B. (1981) “A randomized four- years study of periodontal therapy," Journal of Periodontology, 52, 227-242.

30.Rabbani, G.M., Ash, M.M.Jr. and Caffesse, R.G. (1981) "The effectiveness of subgingival scaling and root planing in calculus removal," Journal of Periodontology, 52, 119-123.

31.Rassameemasmaung, S., Sirirat, M., Komwatchara, T., Rojanapanthu, P., Yudhasaraprasithi, S., Amornchat, C., Gritsanapan, W. and Saraya, A. (1998) "Subgingival administration of Andrographis paniculata gel as an adjunct in the treatment of adult periodontitis," Mahidol Journal, 5, 9-15.

32.Saglie, F.R., Carranza, F.A.Jr, Newman, M.G., Cheng, L. and Lewin, K.J. (1982) "Identification of tissue-invading bacteria in human periodontal disease," Journal of Periodontal Research, 17, 452-455.

33.Silness, J. and Löe, H. (1964) "Periodontal Disease in Pregnancy. II. Correlation between Oral Hygiene and Periodontal Condition," Acta Odontologica Scandinavica, 22, 121-135.

34.Sirirat, M. and Rojanapanthu, P. (2003) "The adjunctive use of Andrographis paniculata gel in periodontal treatment: report of 3 cases," Thai Journal Periodontology, 1, 44-53.

35.Thawornrungroj, S., Kuphasuk, Y., Petmitr, S., Srisatjaluk, R. and Kitkumthorn, N. (2011) "The application of Andrographis paniculata gel as an adjunct in the treatment of chronic periodontitis: clinical and microbiological effects," Naresuan University Journal, 19(2), 38-49.

36.Waerhaug, J. (1978) "Healing of the dento-epithelial junction following subgingival plaque control. II: As observed on extracted teeth," Journal of Periodontology, 49, 119-134. 